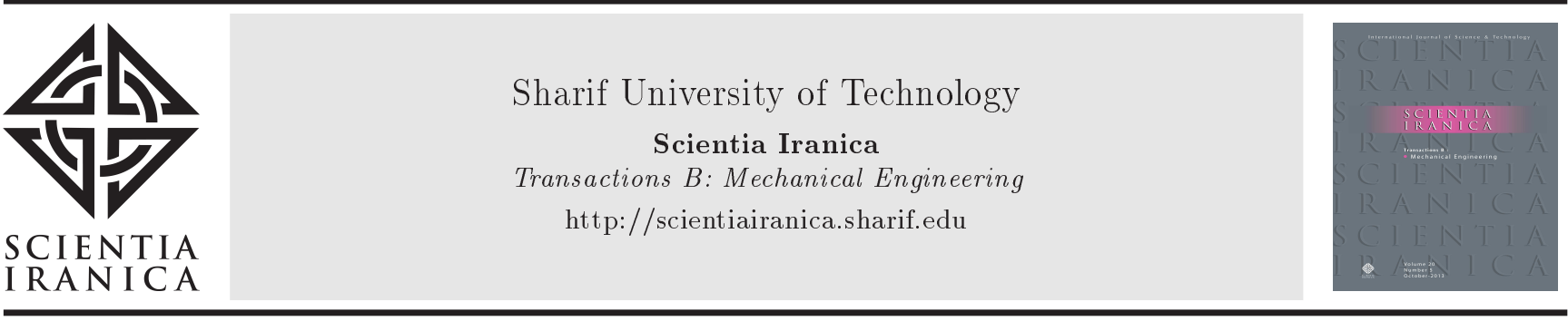

Research Note

\title{
Comparison of nonlinear filtering techniques for inertial sensors error identification in INS/GPS integration
}

\author{
S. Kaviani, H. Salarieh*, A. Alasty, and M. Abediny \\ School of Mechanical Engineering, Sharif University of Technology, Tehran, P.O. Box 11365-9567, Iran.
}

Received 11 November 2015; received in revised form 2 September 2016; accepted 17 April 2017

\author{
KEYWORDS \\ Extended Kalman \\ Filter (EKF); \\ Unscented Kalman \\ Filter (UKF); \\ Extended Particle \\ Filter (EPF); \\ Unscented Particle \\ Filter (UPF).
}

\begin{abstract}
Nonlinear filtering techniques are used to fuse the Global Positioning System (GPS) with Inertial Navigation System (INS) to provide a robust and reliable navigation system with a performance superior to that of either INS or GPS alone. Prominent nonlinear estimators in this field are Kalman Filters (KF) and Particle Filters (PF). The main objective of this research is the comparative study of the well-established filtering methods of EKF, UKF, and PF based on EKF and UKF in an INS-GPS integrated navigation system. Different features of INS-GPS integrated navigation methods in the state estimation, bias estimation, and bias/scale factor estimation are investigated using these four filtering algorithms. Both ground-vehicle experimental test and flight simulation test have been utilized to evaluate the filters performance.
\end{abstract}

(C) 2018 Sharif University of Technology. All rights reserved.

\section{Introduction}

The global positioning system can determine an observer's position on Earth with acceptable accuracy using four or more satellites; however, sometimes, GPS signal obstruction can occur due to signal blockage, multipath effects, interference, or jamming. To overcome the limitations of GPS, the inertial navigation system can be integrated with GPS. In INS, measurements from accelerometer and gyroscope are used to determine the position and orientation of an object. However, the errors of INS increase without bounds in the long term due to inherent sensors errors. It can be said that INS and GPS have complementary

\footnotetext{
*. Corresponding author. Tel: 02166165538 ;

Fax: 02166000021

E-mail addresses: samirakaviani@alum.sharif.edu (S.

Kaviani); salarieh@sharif.edu (H. Salarieh);

alasti@sharif.edu (A. Alasty); mabedinyna@yahoo.com ( $M$. Abediny)
}

doi: $10.24200 /$ sci. 2017.4328 error characteristics; therefore, fusion of INS with GPS results in a more accurate, reliable and continuous positioning solution. The most common sensor fusion technique is Extended Kalman Filter (EKF). This filter linearizes the primary nonlinear state model around the previous estimated state vector, and this technique may show divergence problems due to the linearization process in some applications. Alternatively, another KF type, named Unscented Kalman Filter (UKF), uses a fixed number of sample points to avoid the linearization of the original nonlinear model. Nevertheless, UKF algorithm still treats the noise as a Gaussian distribution [1].

Particle filter is another fusion technique that can deal with any nonlinearities or distributions without any assumptions on the system model and noise distribution. In fact, PFs are sequential Monte Carlo methods based on which particles are drawn from the proposal density function to evaluate the probability density function of the system to estimate the system state variables. A full description of different filtering techniques is provided in [2]. In PFs, choosing the 
proposal density function from which samples are drawn is one of the most important issues. One method to determine the proposal density function is to use other filters' approximations, e.g. EKF and UKF, as the proposal density functions [3,4]. There are several examples of different filtering techniques used in INS-GPS navigation systems. For instance, Wendel and Trommer [5] used a tightly coupled INSGPS integration for missile applications and, in [6], data from GPS, IMU, and a digital compass were fused together using UKF algorithm. In [7], the investigators used EKF to fuse GPS with IMU data, and in [8], both EKF and UKF algorithms were applied onto INSGPS integration using quaternions as a representation of the attitudes. In addition, some adaptive approaches to KF were proposed toward INS-GPS navigation systems [9,10]. In [11-14], the authors proposed different algorithms of the PFs, including UPF and EPF, for INS-GPS integrated systems. Li, et al. [15] carried out both simulation test and vehicle-ground experiment to compare the performances of the direct and indirect modes in an INS-GPS integrated system. Moreover, some recent researches, such as [16-19], on INS-GPS navigation system started to focus on the application of other techniques such as neural networks, learning algorithms, and map matching along with the fusion algorithms. The main contribution of this paper is the comparative study of the well-established filtering methods of EKF, UKF, EPF, and UPF. Different features of integration methods in the state estimation, bias estimation, and bias/scale factor estimation in the indirect mode of INS-GPS integration are investigated using these four filtering methods. The accuracy and robustness of these algorithms are verified by carrying out both ground-vehicle experimental and flight simulation tests. The paper is organized as follows. Section 2 will introduce the theory and implementation details of PFs. Section 3 discusses INS-GPS indirect nonlinear state models in three different state models. Section 4 discusses results, and Section 5 provides the concluding remarks and future work.

\section{Particle filtering}

Particle Filters (PFs) which calculate sequential Monte Carlo estimations numerically through a set of random particles are suboptimal filters. Depending on which proposal density functions or resampling methods are chosen, different versions of PFs are made. The state model is assumed as follows:

$$
\begin{aligned}
& x_{k+1}=f\left(x_{k}, v_{k}\right), \\
& z_{k}=h\left(x_{k}, n_{k}\right) .
\end{aligned}
$$

Sampling Importance Resampling (SIR) Particle Filter, Unscented Particle Filter (UPF), and Extended Particle Filter (EPF) are summarized as follows. The symbols used in this section are given in Table 1.

\subsection{Sampling Importance Resampling (SIR) particle filter}

This basic version of PF uses the transitional density function as the proposal density function. In other words, particles are predicted from the motion model, and the received measurements are used to compute the particles' importance weights. It is worth mentioning that as the proposal density function is independent of the observation likelihood, this filter can be inefficient and sensitive to outliers:

$$
q\left(x_{k} \mid x_{k-1}^{i}, z_{k}\right)=p\left(x_{k} \mid x_{k-1}^{i}\right) .
$$

The pseudo code for SIR particle filter is summarized below $[3,4]$.

\section{Pseudo code for SIR particle filter}

i. Initialization: random generation of $N$ particles from the initial distribution $p\left(x_{0}\right)$ :

Table 1. List of symbols in the filters.

\begin{tabular}{cc}
\hline$q\left(x_{k} \mid x_{k-1}, z_{k}\right)$ & Proposal density function \\
$p\left(x_{k} \mid x_{k-1}\right)$ & Transitional density function \\
$p\left(z_{k} \mid x_{k}\right)$ & Likelihood function \\
$N(x ; m, P)$ & Gaussian density with argument $x$, mean $m$, and covariance $P$ \\
$n_{x}$ & Dimension of the state vector \\
$n_{v}$ & Dimension of the process noise \\
$n_{n}$ & Dimension of the measurement noise \\
$\alpha$ & Scaling parameters in the unscented transformation \\
$\beta$ &
\end{tabular}




$$
\left[\begin{array}{c}
x_{0}^{i} \sim p\left(x_{0}\right) \quad i=1, \ldots, N \\
w_{0}^{i}=\frac{1}{N}
\end{array}\right.
$$

for $k=1,2, \ldots$

ii. Importance sampling step for $i=1,2, \ldots, N$ :

$$
x_{k}^{i} \sim q\left(x_{k} \mid x_{k-1}^{i}, z_{k}\right)=p\left(x_{k} \mid x_{k-1}^{i}\right) .
$$

iii. Evaluation of the particles' importance weights for $i=1,2, \ldots, N$ :

$$
\begin{aligned}
w_{k}^{i} & =w_{k-1}^{i} \frac{p\left(z_{k} \mid x_{k}^{i}\right) p\left(x_{k}^{i} \mid x_{k-1}^{i}\right)}{q\left(x_{k}^{i} \mid x_{k-1}^{i}, z_{k}\right)} \\
& =w_{k-1}^{i} p\left(z_{k} \mid x_{k}^{i}\right) .
\end{aligned}
$$

iv. Normalization of the importance weights for $i=$ $1,2, \ldots N$ :

$$
w_{k}^{i}=w_{k}^{i}\left(\sum_{j=1}^{N} w_{k}^{j}\right)^{-1} .
$$

v. Resampling step, obtaining $N$ samples by suppression of the low importance weighted samples and multiplication of the high importance weighted samples, and assigning equal weights to the samples:

$$
\left(x_{k}^{i}, w_{k}^{i}\right) \stackrel{\text { Resampling }}{\Rightarrow}\left(x_{k}^{i}, w_{k}^{i}=\frac{1}{N}\right) .
$$

iv. Output: Computation of desired statistical measures, such as mean and covariance, using a set of selected samples:

$$
\begin{aligned}
& \bar{X}_{k}=\frac{1}{N} \sum_{j=1}^{N} x_{k}^{j}, \\
& \bar{P}_{k}=\frac{1}{N} \sum_{j=1}^{N}\left(\bar{X}_{k}-x_{k}^{j}\right)\left(\bar{X}_{k}-x_{k}^{j}\right)^{T} .
\end{aligned}
$$

\subsection{Unscented particle filter and extended particle filter}

As mentioned earlier, in these versions of $\mathrm{PF}$, the proposal density function is devised by UKF, EKF, or Gaussian approximations. In fact, in order to sample particles from the region of high likelihood, EPF and UPF use EKF and UKF to generate the proposal density function for each individual particle. The description for EPF and UPF is presented according to $[3,4]$.

\section{Pseudo code for extended particle filter}

i. Initialization: random generation of $N$ particles from initial distribution $p\left(x_{0}\right)$ :

$$
x_{0}^{i} \sim p\left(x_{0}\right) \quad w_{0}^{i}=\frac{1}{N} \quad i=1, \ldots, N
$$

for $k=1,2, \ldots$

ii. For $i=1,2, \ldots, N$ :

$$
\begin{aligned}
& \bar{x}_{k \mid k-1}^{(i)}=f\left(x_{k-1}^{(i)}\right) \\
& P_{k \mid k-1}^{(i)}=F_{k-1}^{(i)} P_{k-1}^{(i)} F_{k-1}^{T(i)}+G_{k-1}^{(i)} Q_{k-1} G_{k-1}^{T(i)} \\
& F_{k-1}^{(i)}=\left.\frac{\partial f_{k-1}}{\partial x}\right|_{x_{k-1}^{(i)}}, \\
& G_{k-1}^{(i)}=\left.\frac{\partial f_{k-1}}{\partial v}\right|_{x_{k-1}^{(i)}}, \\
& H_{k}^{(i)}=\left.\frac{\partial h_{k}}{\partial x}\right|_{\bar{x}_{k \mid k-1}^{(i)}}, U_{k}^{(i)}=\left.\frac{\partial h_{k}}{\partial n}\right|_{\bar{x}_{k \mid k-1}^{(i)}} \\
& K_{k}=P_{k \mid k-1}^{(i)} H_{k}^{T(i)}\left[U_{k}^{(i)} R_{k} U_{k}^{T(i)}\right. \\
& \left.\quad+H_{k}^{(i)} P_{k \mid k-1}^{(i)} H_{k}^{T(i)}\right]^{-1} \\
& \bar{x}_{k}^{(i)}=\bar{x}_{k \mid k-1}^{(i)}+K_{k}\left(z_{k}-h\left(\bar{x}_{k \mid k-1}^{(i)}\right)\right) \\
& \hat{P}_{k}^{(i)}=P_{k \mid k-1}^{(i)}-K_{k} H_{k}^{(i)} P_{k \mid k-1}^{(i)} \cdot
\end{aligned}
$$

Importance sampling:

$$
\hat{x}_{k}^{(i)} \sim q\left(x_{k}^{(i)} \mid x_{k-1}^{(i)}, z_{k}\right)=N\left(\bar{x}_{k}^{(i)}, \hat{P}_{k}^{(i)}\right) .
$$

iii. Evaluation of the importance weights for $i=$ $1,2, \ldots, N$ :

$$
\begin{aligned}
\bar{W}_{k}^{i} \propto \frac{p\left(z_{k} \mid \hat{x}_{k}^{(i)}\right) p\left(\hat{x}_{k}^{(i)} \mid x_{k-1}^{(i)}\right)}{q\left(\hat{x}_{k}^{(i)} \mid x_{k-1}^{(i)}, z_{k}\right)} \\
=\frac{p\left(z_{k} \mid \hat{x}_{k}^{(i)}\right) p\left(\hat{x}_{k}^{(i)} \mid x_{k-1}^{(i)}\right)}{N\left(\hat{x}_{k}^{(i)} ; \bar{x}_{k}^{(i)}, \hat{P}_{k}^{(i)}\right)} \\
=\frac{p\left(z_{k} \mid \hat{x}_{k}^{(i)}\right) N\left(\hat{x}_{k}^{(i)} ; \bar{x}_{k \mid k-1}^{(i)}, P_{k \mid k-1}^{(i)}\right)}{N\left(\hat{x}_{k}^{(i)} ; \bar{x}_{k}^{(i)}, \hat{P}_{k}^{(i)}\right)} .
\end{aligned}
$$

iv. Normalization of the importance weights for $i=$ $1,2, \ldots, N$ :

$$
W_{k}^{i}=\bar{W}_{k}^{i}\left(\sum_{j=1}^{N} \bar{W}_{k}^{j}\right)^{-1} .
$$


v. Resampling step, obtaining $N$ samples by suppression of the low importance weighted samples and multiplication of the high importance weighted samples, and assigning equal weights to the samples:

$$
\left(\hat{x}_{k}^{(i)}, \hat{P}_{k}^{(i)}, W_{k}^{i}\right) \stackrel{\text { Resampling }}{\Rightarrow}\left(x_{k}^{(i)}, P_{k}^{(i)}, W_{k}^{i}=\frac{1}{N}\right)_{(20)}
$$

vi. Calculation of outputs:

$$
\begin{aligned}
& \bar{X}_{k}=\frac{1}{N} \sum_{i=1}^{N} x_{k}^{(i)}, \\
& \bar{P}_{k}=\frac{1}{N} \sum_{i=1}^{N}\left(\bar{X}_{k}-x_{k}^{(i)}\right)\left(\bar{X}_{k}-x_{k}^{(i)}\right)^{T} .
\end{aligned}
$$

\section{Pseudo code for unscented particle filter}

i. Initialization: Random generation of $N$ particles from initial distribution, $p\left(x_{0}\right)$, and setting of the augmented state vector and covariance for $i=$ $1,2, \ldots, N$ :

$$
\begin{aligned}
& x_{0}^{(i) a}=\left[\left(x_{0}^{(i)}\right)^{T} 00\right]^{T}, \\
& P_{0}^{(i) a}=\left[\begin{array}{ccc}
P_{0}^{(i)} & 0 & 0 \\
0 & Q & 0 \\
0 & 0 & R
\end{array}\right],
\end{aligned}
$$

$$
\text { for } k=1,2, \ldots
$$

ii. For $i=1,2, \ldots, N$ :

Sigma points calculation:

$$
\begin{aligned}
& \mathcal{X}_{k-1}^{(i) a}=\left[x_{k-1}^{(i) a} x_{k-1}^{(i) a} \pm \sqrt{\left(n_{a}+\lambda\right) P_{k-1}^{(i) a}}\right] \\
& n_{a}=n_{x}+n_{v}+n_{n}, \\
& \mathcal{X}_{k \mid k-1}^{(i) x}=f\left(\mathcal{X}_{k-1}^{(i) x}, \mathcal{X}_{k-1}^{(i) v}\right), \\
& \bar{x}_{k \mid k-1}^{(i)}=\sum_{j=0}^{2 n_{a}} W_{j}^{(m)} \mathcal{X}_{j, k \mid k-1}^{(i) x}, \quad W_{0}^{(m)}=\frac{\lambda}{n_{a}+\lambda}, \\
& W_{0}^{(c)}=\frac{\lambda}{n_{a}+\lambda}+\left(1-\alpha^{2}+\beta\right), \\
& W_{j}^{(m)}=W_{j}^{(c)}=\frac{1}{2\left(n_{a}+\lambda\right)}, \\
& \lambda=\alpha^{2}\left(n_{a}+\kappa\right)-n_{a} .
\end{aligned}
$$

$$
\begin{aligned}
& P_{k \mid k-1}^{(i)}= \sum_{j=0}^{2 n_{a}} W_{j}^{(c)}\left[\mathcal{X}_{j, k \mid k-1}^{(i) x}-\bar{x}_{k \mid k-1}^{(i)}\right] \\
& {\left[\mathcal{X}_{j, k \mid k-1}^{(i) x}-\bar{x}_{k \mid k-1}^{(i)}\right]^{T}, } \\
& \mathcal{Y}_{k \mid k-1}^{(i)}= h\left(\mathcal{X}_{k \mid k-1}^{(i) x}, \mathcal{X}_{k-1}^{(i) n}\right), \\
& \bar{y}_{k \mid k-1}^{(i)}= \sum_{j=0}^{2 n_{a}} W_{j}^{(m)} \mathcal{Y}_{j, k \mid k-1}^{(i)}, \\
& P_{y_{k} y_{k}=}=\sum_{j=0}^{2 n_{a}} W_{j}^{(c)}\left[\mathcal{Y}_{j, k \mid k-1}^{(i)}-\bar{y}_{k \mid k-1}^{(i)}\right] \\
& {\left[\mathcal{Y}_{j, k \mid k-1}^{(i)}-\bar{y}_{k \mid k-1}^{(i)}\right]^{T}, } \\
& P_{x_{k} y_{k}=}=\sum_{j=0}^{2 n_{a}} W_{j}^{(c)}\left[\mathcal{X}_{j, k \mid k-1}^{(i) x}-\bar{x}_{k \mid k-1}^{(i)}\right] \\
& \hat{P}_{k}^{(i)}=P_{k \mid k-1}^{(i)}-K_{k} P_{y_{k} y_{k}} K_{k}^{T} . \\
& K_{k}=P_{x_{k} y_{k}}^{(i)} P_{y_{k} y_{k}}^{-1}, \\
& \bar{x}_{k}^{i}=\bar{x}_{k \mid k-1}^{(i)}+K_{k}\left(z_{k}-\bar{y}_{k \mid k-1}^{(i)}\right), \\
&\left.\hat{y}_{k \mid k-1}\right]^{T},
\end{aligned}
$$

Importance sampling:

$$
\hat{x}_{k}^{(i)} \sim q\left(x_{k}^{(i)} \mid x_{k-1}^{(i)}, z_{k}\right)=N\left(\bar{x}_{k}^{(i)}, \hat{P}_{k}^{(i)}\right) .
$$

iii. Evaluation of the importance weights for $i=$ $1,2, \ldots, N$ :

$$
\begin{aligned}
\bar{W}_{k}^{i} \propto \frac{p\left(z_{k} \mid \hat{x}_{k}^{(i)}\right) p\left(\hat{x}_{k}^{(i)} \mid x_{k-1}^{(i)}\right)}{q\left(\hat{x}_{k}^{(i)} \mid x_{k-1}^{(i)}, z_{k}\right)} \\
=\frac{p\left(z_{k} \mid \hat{x}_{k}^{(i)}\right) p\left(\hat{x}_{k}^{(i)} \mid x_{k-1}^{(i)}\right)}{N\left(\hat{x}_{k}^{(i)} ; \bar{x}_{k}^{(i)}, \hat{P}_{k}^{(i)}\right)} \\
=\frac{p\left(z_{k} \mid \hat{x}_{k}^{(i)}\right) N\left(\hat{x}_{k}^{(i)} ; \bar{x}_{k \mid k-1}^{(i)}, P_{k \mid k-1}^{(i)}\right)}{N\left(\hat{x}_{k}^{(i)} ; \bar{x}_{k}^{(i)}, \hat{P}_{k}^{(i)}\right)} .
\end{aligned}
$$

iv. Normalization of the importance weights for $i=$ $1,2, \ldots N$ :

$$
W_{k}^{i}=\bar{W}_{k}^{i}\left(\sum_{j=1}^{N} \bar{W}_{k}^{j}\right)^{-1} .
$$


v. Resampling step, obtaining $N$ samples by suppression of the low importance weighted samples and multiplication of the high importance weighted samples, and assigning equal weights to the samples:

$$
\left(\hat{x}_{k}^{(i)}, \hat{P}_{k}^{(i)}, W_{k}^{i}\right) \stackrel{\text { Resampling }}{\Rightarrow}\left(x_{k}^{(i)}, P_{k}^{(i)}, W_{k}^{i}=\frac{1}{N}\right)_{(39)}
$$

vi. Calculation of output:

$$
\begin{aligned}
& \bar{X}_{k}=\frac{1}{N} \sum_{i=1}^{N} x_{k}^{(i)}, \\
& \bar{P}_{k}=\frac{1}{N} \sum_{i=1}^{N}\left(\bar{X}_{k}-x_{k}^{(i)}\right)\left(\bar{X}_{k}-x_{k}^{(i)}\right)^{T} .
\end{aligned}
$$

\section{INS-GPS system and measurement model}

The navigation equations are developed on the basis of NED navigation frame, which is widely used in the navigation applications. The error dynamics equations obtained using perturbation analysis are presented in three types of models: 9-variable, 15-variable, and 21variable models. The perturbation of the position, velocity, attitude, and gravity can be expressed as [20,21].

\section{Equation section}

$$
\begin{aligned}
& \hat{r}^{n}=r^{n}+\delta r^{n}, \\
& \hat{v}^{n}=v^{n}+\delta v^{n}, \\
& \hat{C}_{b}^{n}=\left(I_{3 \times 3}-E^{n}\right) C_{b}^{n}, \\
& E^{n}=\left[\begin{array}{ccc}
0 & -\varepsilon_{D} & \varepsilon_{E} \\
\varepsilon_{D} & 0 & -\varepsilon_{N} \\
-\varepsilon_{E} & \varepsilon_{N} & 0
\end{array}\right],
\end{aligned}
$$

$$
\hat{g}^{n}=g^{n}+\delta g^{n}
$$

where $\Lambda$ and $\delta$ denote computed values and errors. $E^{n}$ denotes the skew symmetric form of the attitude errors, and $N, E$, and $D$ refer to the north, east, and down directions. The symbols used in this section are given in Table 2.

\subsection{9-state-variable system model}

Without considering the inertial sensors' error, the error dynamics equations are obtained in the nonlinear form as shown in Box I, where $\delta f_{k-1}^{b}$ and $\delta \omega_{i b}^{b}$ are the process noise vector associated with each of the IMU measurements. In other words, part of the stochastic errors in the readings of the accelerometer triad is $\delta f_{k-1}^{b}$ and part of the stochastic errors in the readings of the gyroscope triad is $\delta \omega_{i b}^{b}$ :

$$
\begin{aligned}
& \hat{f}_{b}=f_{b}+\delta f_{b}=\left[\begin{array}{c}
f_{b}^{x} \\
f_{b}^{y} \\
f_{b}^{z}
\end{array}\right]+\left[\begin{array}{l}
\delta f_{b}^{x} \\
\delta f_{b}^{y} \\
\delta f_{b}^{z}
\end{array}\right], \\
& \hat{\omega}_{i b}^{b}=\omega_{i b}^{b}+\delta \omega_{i b}^{b}=\left[\begin{array}{c}
\omega_{i b}^{b x} \\
\omega_{i b}^{b y} \\
\omega_{i b}^{b z}
\end{array}\right]+\left[\begin{array}{l}
\delta \omega_{i b}^{b x} \\
\delta \omega_{i b}^{b y} \\
\delta \omega_{i b}^{b z}
\end{array}\right] .
\end{aligned}
$$

\begin{tabular}{|c|c|c|c|}
\hline$\varphi$ & Latitude & $\delta r$ & Position error vector \\
\hline$\lambda$ & Longitude & $\delta v$ & Velocity error vector \\
\hline$h$ & Altitude & $\varepsilon$ & Attitude error vector \\
\hline $\begin{array}{l}V_{N} \\
V_{E} \\
V_{D}\end{array}$ & Velocity components along north, east, and down & $\omega_{i b}^{b}$ & Gyroscope output vector \\
\hline$M$ & Meridian radius of curvature of the earth reference ellipsoid & $f_{b}$ & Accelerometer output vector \\
\hline$N$ & Normal radius of curvature of the earth reference ellipsoid & $b_{\text {acc }}$ & Accelerometer bias vector \\
\hline$C_{b}^{n}$ & Direction cosine matrix from the body to navigation frame & $b_{\mathrm{gyr}}$ & Gyroscope bias vector \\
\hline$\omega_{i e}^{n}$ & $\begin{array}{l}\text { Rotation rate vector of the earth frame with respect to the } \\
\text { inertial frame projected to the navigation frame }\end{array}$ & $\varrho_{\text {acc }}$ & Accelerometer scale factor vector \\
\hline$\omega_{e n}^{n}$ & $\begin{array}{c}\text { Rotation rate vector of the navigation frame with respect to the } \\
\text { earth frame projected to the navigation frame }\end{array}$ & $\varrho_{\mathrm{gyr}}$ & Gyroscope scale factor vector \\
\hline
\end{tabular}

\subsection{5-state-variable system model}

In this model, it is assumed that the outputs measured by accelerometer triad and gyroscope triad consist of the constant bias and zero mean white noise:

$$
\begin{aligned}
\hat{f}_{b}=f_{b} & +b_{a c c}+\delta f_{b}=\left[\begin{array}{l}
f_{b}^{x} \\
f_{b}^{y} \\
f_{b}^{z}
\end{array}\right]+\left[\begin{array}{l}
b_{a c c}^{x} \\
b_{a c c}^{y} \\
b_{a c c}^{z}
\end{array}\right] \\
& +\left[\begin{array}{l}
\delta f_{b}^{x} \\
\delta f_{b}^{y} \\
\delta f_{b}^{z}
\end{array}\right]
\end{aligned}
$$

Table 2. List of symbols in the equations. 


$$
\begin{aligned}
& F_{r r}=\left[\begin{array}{ccc}
0 & 0 & \frac{-V_{N}}{(M+h)^{2}} \\
\frac{V_{E} \sin \varphi}{(N+h)(\cos \varphi)^{2}} & 0 & \frac{-V_{E}}{(N+h)^{2} \cos \varphi} \\
0 & 0 & 0
\end{array}\right] \\
& F_{r v}=\left[\begin{array}{ccc}
\frac{1}{M+h} & 0 & 0 \\
0 & \frac{1}{(N+h) \cos \varphi} & 0 \\
0 & 0 & -1
\end{array}\right]
\end{aligned}
$$

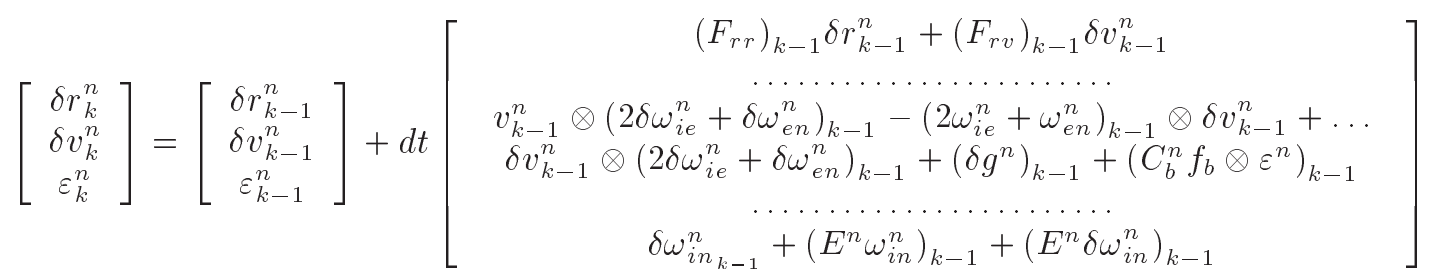

$$
\begin{aligned}
& +d t\left[\begin{array}{c}
0_{3 \times 1} \\
\left(C_{b}^{n}-E^{n} C_{b}^{n}\right)_{k-1} \delta f_{k-1}^{b} \\
-C_{b_{k-1}}^{n}\left(\delta \omega_{i b}^{b}\right)_{k-1}
\end{array}\right] .
\end{aligned}
$$

Box I

$$
\begin{aligned}
\hat{\omega}_{i b}^{b}= & \omega_{i b}^{b}+b_{g y r}+\delta \omega_{i b}^{b}=\left[\begin{array}{c}
\omega_{i b}^{b x} \\
\omega_{i b}^{b y} \\
\omega_{i b}^{b z}
\end{array}\right]+\left[\begin{array}{c}
b_{g y r}^{x} \\
b_{g y r}^{y} \\
b_{g y r}^{z}
\end{array}\right] \\
& +\left[\begin{array}{c}
\delta \omega_{i b}^{b x} \\
\delta \omega_{i b}^{b y} \\
\delta \omega_{i b}^{b z}
\end{array}\right] .
\end{aligned}
$$

With this assumption, the error dynamics equations are obtained by Eq. (54) as shown in Box II.

\subsection{1-state-variable system model}

In this model, it is assumed that the outputs of the IMU consist of the scale factor, constant bias, and zero mean white noise as follows:

$$
\begin{aligned}
\hat{f}_{b} & =\left[\begin{array}{c}
S_{a c c}^{x} f_{b}^{x} \\
S_{a c c}^{y} f_{b}^{y} \\
S_{a c c}^{z} f_{b}^{z}
\end{array}\right]+\left[\begin{array}{c}
b_{a c c}^{x} \\
b_{a c c}^{y} \\
b_{a c c}^{z}
\end{array}\right]+\left[\begin{array}{l}
\delta f_{b}^{x} \\
\delta f_{b}^{y} \\
\delta f_{b}^{z}
\end{array}\right] \\
& =\left[\begin{array}{c}
\left(1+\varrho_{a c c}^{x}\right) f_{b}^{x} \\
\left(1+\varrho_{a c c}^{y}\right) f_{b}^{y} \\
\left(1+\varrho_{a c c}^{z}\right) f_{b}^{z}
\end{array}\right]+\left[\begin{array}{c}
b_{a c c}^{x} \\
b_{a c c}^{y} \\
b_{a c c}^{z}
\end{array}\right]+\left[\begin{array}{c}
\delta f_{b}^{x} \\
\delta f_{b}^{y} \\
\delta f_{b}^{z}
\end{array}\right],
\end{aligned}
$$

$$
\begin{aligned}
\hat{\omega}_{i b}^{b} & =\left[\begin{array}{c}
S_{g y r}^{x} \omega_{i b}^{b x} \\
S_{g y r}^{x} \omega_{i b}^{b y} \\
S_{g y r}^{x} \omega_{i b}^{b z}
\end{array}\right]+\left[\begin{array}{c}
b_{g y r}^{x} \\
b_{g y r}^{y} \\
b_{g y r}^{z}
\end{array}\right]+\left[\begin{array}{c}
\delta \omega_{i b}^{b x} \\
\delta \omega_{i b}^{b y} \\
\delta \omega_{i b}^{b z}
\end{array}\right] \\
& =\left[\begin{array}{c}
\left(1+\varrho_{g y r}^{x}\right) \omega_{i b}^{b x} \\
\left(1+\varrho_{g y r}^{y}\right) \omega_{i b}^{b y} \\
\left(1+\varrho_{g y r}^{z}\right) \omega_{i b}^{b z}
\end{array}\right]+\left[\begin{array}{c}
b_{g y r}^{x} \\
b_{g y r}^{y} \\
b_{g y r}^{z}
\end{array}\right]+\left[\begin{array}{c}
\delta \omega_{i b}^{b x} \\
\delta \omega_{i b}^{b y} \\
\delta \omega_{i b}^{b z}
\end{array}\right] .
\end{aligned}
$$

On the basis of this assumption, the error dynamics equations are given in Eq. (57) as shown in Box III.

\subsection{Measurement model}

The position and velocity from GPS can be considered as measurements. The measurement equation can be written as follows:

$$
\begin{aligned}
& z_{k}=\left(\begin{array}{c}
r_{\mathrm{INS}}^{n}-r_{\mathrm{GPS}}^{n} \\
v_{\mathrm{INS}}^{n}-v_{\mathrm{GPS}}^{n}
\end{array}\right)=\left(\begin{array}{c}
\varphi_{\mathrm{INS}}^{n}-\varphi_{\mathrm{GPS}}^{n} \\
\lambda_{\mathrm{INS}}^{n}-\lambda_{\mathrm{GPS}}^{n} \\
h_{\mathrm{INS}}^{n}-h_{\mathrm{GPS}}^{n} \\
v_{\mathrm{INS}}^{n}-v_{\mathrm{GPS}}^{n}
\end{array}\right), \\
& z_{k}=H x_{k}+e_{k}, \\
& e_{k} \sim N\left(0, R_{k}\right),
\end{aligned}
$$

$\varphi$ and $\lambda$ are in radians, and they are very small in comparison to the other state variables. Therefore, to 


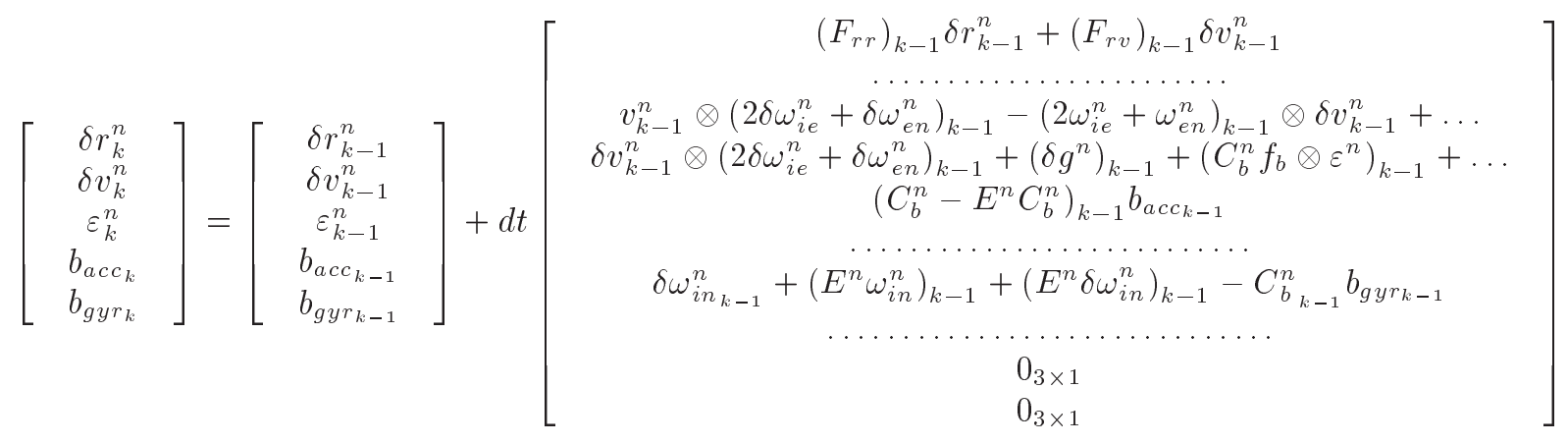

$$
\begin{aligned}
& +d t\left[\begin{array}{c}
0_{3 \times 1} \\
\left(C_{b}^{n}-E^{n} C_{b}^{n}\right)_{k-1} \delta f_{k-1}^{b} \\
-C_{b_{k-1}}^{n}\left(\delta \omega_{i b}^{b}\right)_{k-1} \\
\delta b_{a c c_{k-1}} \\
\delta b_{g y r_{k-1}}
\end{array}\right]
\end{aligned}
$$

\section{Box II}

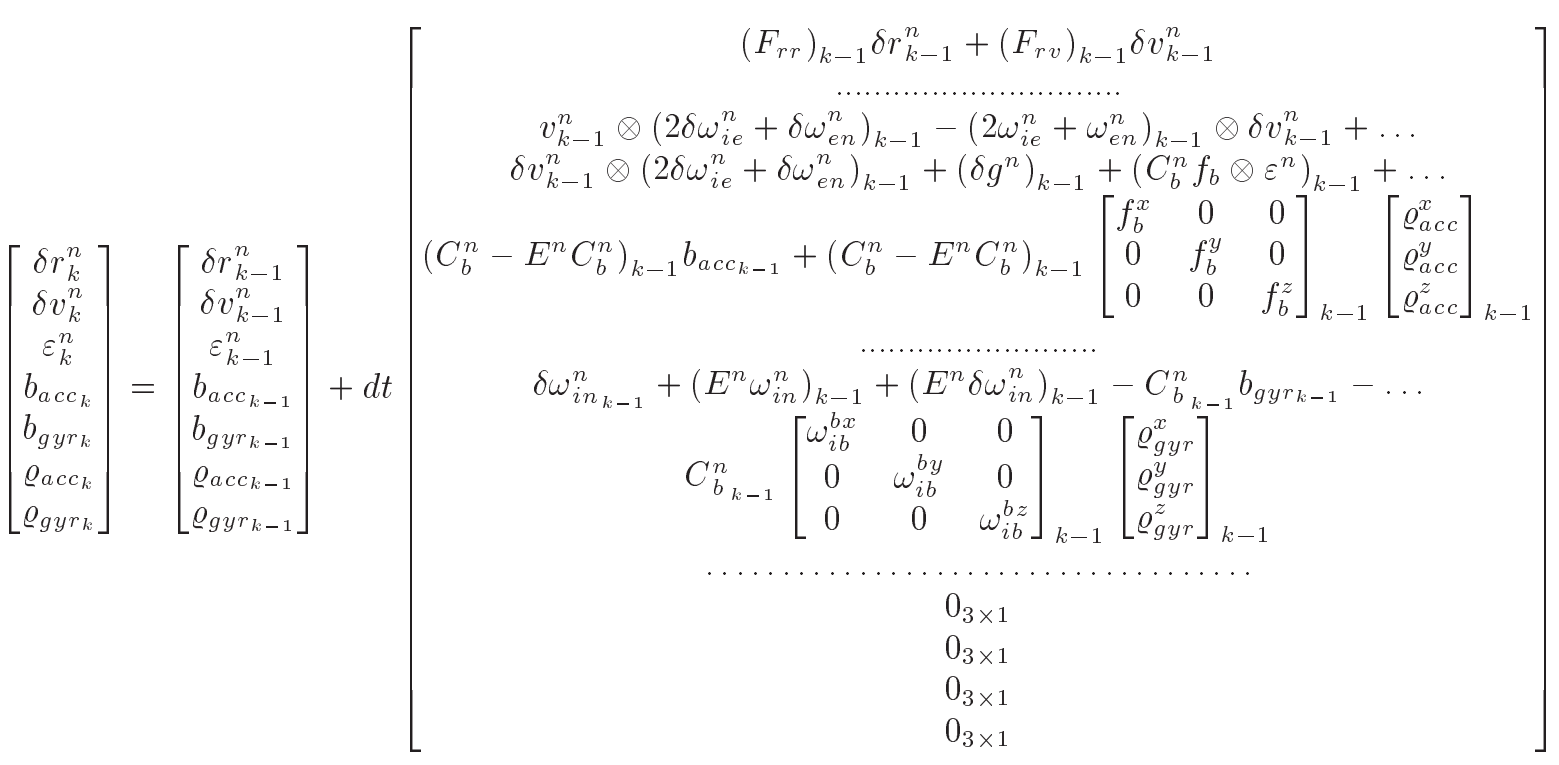

$$
\begin{aligned}
& +d t\left[\begin{array}{c}
0_{3 \times 1} \\
\left(C_{b}^{n}-E^{n} C_{b}^{n}\right)_{k-1} \delta f_{k-1}^{b} \\
-C_{b_{k-1}}^{n}\left(\delta \omega_{i b}^{b}\right)_{k-1} \\
\delta b_{a c c_{k-1}} \\
\delta b_{g y r_{k-1}} \\
\delta \varrho_{a c c_{k-1}} \\
\delta \varrho_{g y r_{k-1}}
\end{array}\right] .
\end{aligned}
$$


avoid numerical issues, $z_{k}$ is taken as follows:

$$
z_{k}=\left(\begin{array}{c}
\left(M+h_{0}\right)\left(\varphi_{\mathrm{INS}}^{n}-\varphi_{\mathrm{GPS}}^{n}\right) \\
\left(N+h_{0}\right) \cos \varphi_{0}\left(\lambda_{\mathrm{INS}}^{n}-\lambda_{\mathrm{GPS}}^{n}\right) \\
\left(h_{\mathrm{INS}}^{n}-h_{\mathrm{GPS}}^{n}\right) \\
v_{\mathrm{INS}}^{n}-v_{\mathrm{GPS}}^{n}
\end{array}\right)
$$

For 9-state-variable system model, $H$ is equal to:

$$
\begin{aligned}
& H= \\
& {\left[\begin{array}{ccccc}
\left(M+h_{0}\right) & 0 & 0 & & \\
0 & \left(N+h_{0}\right) \cos \varphi_{0} & 0 & 0_{3 \times 3} & 0_{3 \times 3} \\
0 & 0 & 1 & & \\
& 0_{3 \times 3} & & I_{3 \times 3} & 0_{3 \times 3}
\end{array}\right] .}
\end{aligned}
$$

For 15-state-variable system model, $H$ is obtained by Eq. (63), as shown in Box IV, and for 21-state-variable system model, it is obtained by Eq. (64) as shown in Box V.

\section{Field test and simulation}

Both ground-vehicle experimental test and flight simulation test are employed to identify inertial sensors' bias and scale factor by different filtering methods including EKF, UKF, EPF, and UPF. The estimation improvement due to identification of inertial sensors' error is shown by comparing the obtained results. An Intel(R) Core (TM) i5-3570 CPU is utilized for integrating GPS and INS.

\subsection{Field test}

A field test lasting $336 \mathrm{~s}$ is conducted based on the car ride. UPF, EPF, UKF, and EKF for all the three presented system models are considered in this test. The road test trajectory and INS estimation of that are shown in Figure 1. In this test, (Gyro VG910F1 Fizoptika FOG) and (Accl QA2000 Honeywell VQ)

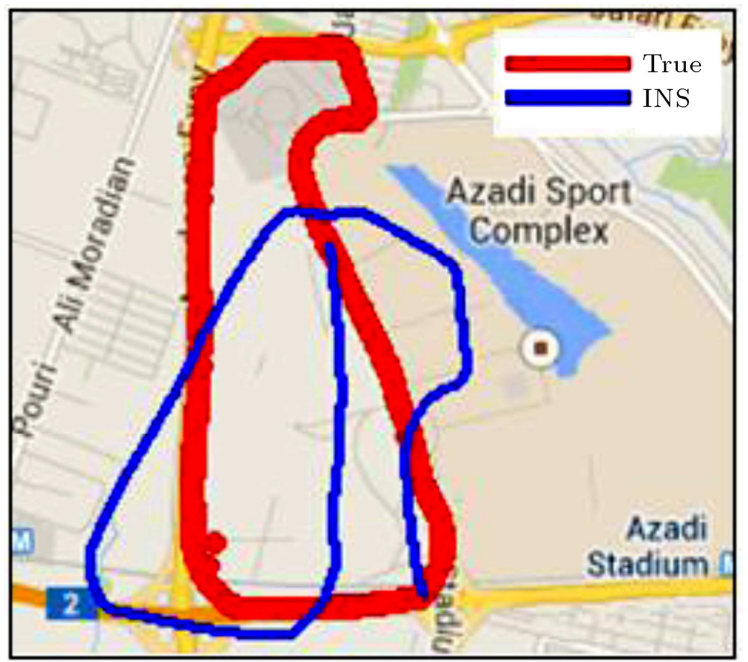

Figure 1. Field test trajectory and INS estimation.

are integrated with the UBlox NEO-6M GPS receiver. The filter is initialized with readings from the magnetometer and accelerometer. The experimental car ride data include 2 sec GPS signal outages as this occurs in reality; however, three to ten seconds GPS signal outages are also purposely assumed for the test, and these outages are shown by yellow bands in figures. Since there is no reference information to evaluate the estimate errors in the performed experimental test, the results are evaluated with respect to a reference solution made by GPS receiver omitting 2 sec GPS signal outages as reference information. In addition, the path of experimental test can be completely specified in a map as shown in Figure 1. However, there is no reference information to evaluate the attitude estimates and inertial sensors' error identification. To overcome these inevitable limitations in our experimental test, we check the algorithms by providing a flight simulation in which the whole true trajectories in position, velocity, and angles are available. The experiment results for all

$$
H=\left[\begin{array}{ccccccc}
\left(M+h_{0}\right) & 0 & 0 & & & & \\
0 & \left(N+h_{0}\right) \cos \varphi_{0} & 0 & 0_{3 \times 3} & 0_{3 \times 3} & 0_{3 \times 3} & 0_{3 \times 3} \\
0 & 0 & 1 & & & & \\
& 0_{3 \times 3} & & I_{3 \times 3} & 0_{3 \times 3} & 0_{3 \times 3} & 0_{3 \times 3}
\end{array}\right] .
$$

\section{Box IV}

$$
H=\left[\begin{array}{ccccccccc}
\left(M+h_{0}\right) & 0 & 0 & & & & & & \\
0 & \left(N+h_{0}\right) \cos \varphi_{0} & 0 & 0_{3 \times 3} & 0_{3 \times 3} & 0_{3 \times 3} & 0_{3 \times 3} & 0_{3 \times 3} & 0_{3 \times 3} \\
0 & 0 & 1 & & & & & & \\
& 0_{3 \times 3} & & I_{3 \times 3} & 0_{3 \times 3} & 0_{3 \times 3} & 0_{3 \times 3} & 0_{3 \times 3} & 0_{3 \times 3}
\end{array}\right]
$$



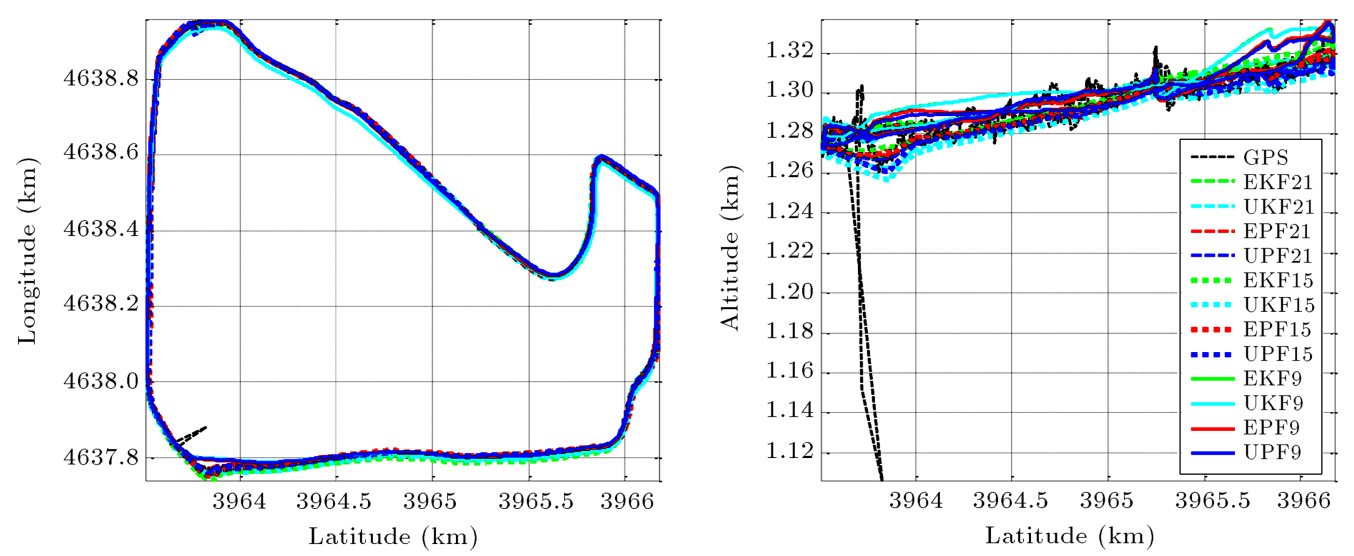

Figure 2. Position estimates for field test.

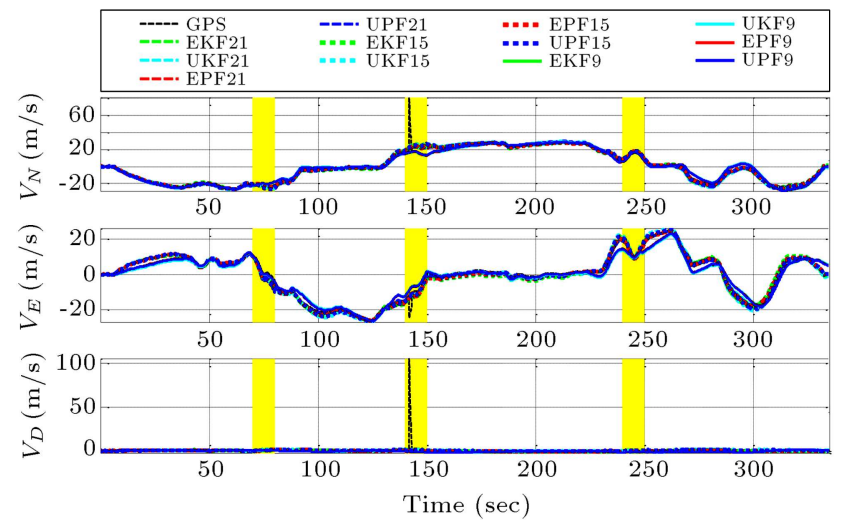

Figure 3. Velocity estimates for field test.

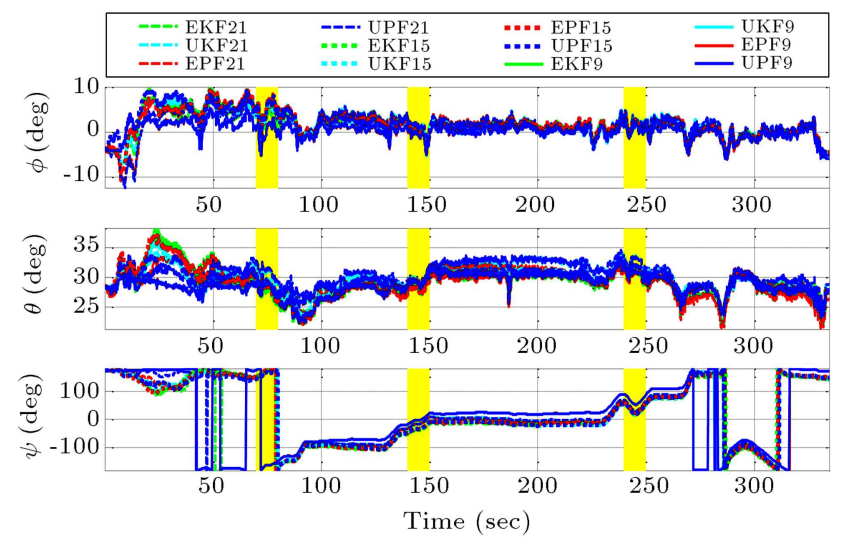

Figure 4. Euler angle estimates for field test.

three types of system models are depicted in Figures 2 to 7 and Table 3 .

In the legends of figures, type of the system model used by the filters is indicated by the number of its state variables. For example, EPF21 refers to EPF employed for 21-state-variable system model. As can be seen in the results, identification of inertial sensors' error resulted in estimates that are more accurate, especially in the 15-state-variable system model for all of the filters. It can be seen that gyro bias estimations have good convergence in comparison to accelerometer bias.
However, there is no reference value for the inertial sensor characteristics to evaluate the accuracy of bias and scale factor estimates. Furthermore, it is shown in Figure 7 that EPF and UPF have much better performances for position estimates in comparison to EKF and UKF for a 9-variable system model.

Number of particles used in UPF and EPF is 15, and the computational time (CPU time) in Table 3 shows that EPF algorithm, similar to EKF and UKF algorithms, can work in real time in 9-variable and 15variable system models.

\subsection{Simulation}

To overcome the experimental test limitations, a flight simulation lasting $800 \mathrm{sec}$ with four $20 \mathrm{sec}$ GPS signal outages, shown by yellow bands in figures, is checked. Inertial sensors' characteristics are illustrated according to Table 4 . Simulation results are available in Figures 8 to 13 and Table 5 .

Similar to the field test results, simulation results showed that identification of inertial sensors' error improved the accuracy of estimates. As seen in Figure 11, the gyroscope biases estimates converged to the true values. In contrast, the accelerometer bias estimates converged to the wrong values, and both gyroscope and accelerometer scale factors were identified poorly. Moreover, EPF and UPF have much better performances for position estimates in comparison to EKF and UKF for all types of system models; performance accuracies of EPF and UPF are similar to those of EKF and UKF for the velocity and attitude estimates of all the three system models.

Number of particles in UPF and EPF is 15, and the computational time shows that EPF algorithm, similar to EKF and UKF algorithms, can work in real time in 9 -variable and 15 -variable system models.

\section{Conclusion}

Within this study, UPF, EPF, UKF, and EKF are implemented for INS-GPS integration during a ground- 


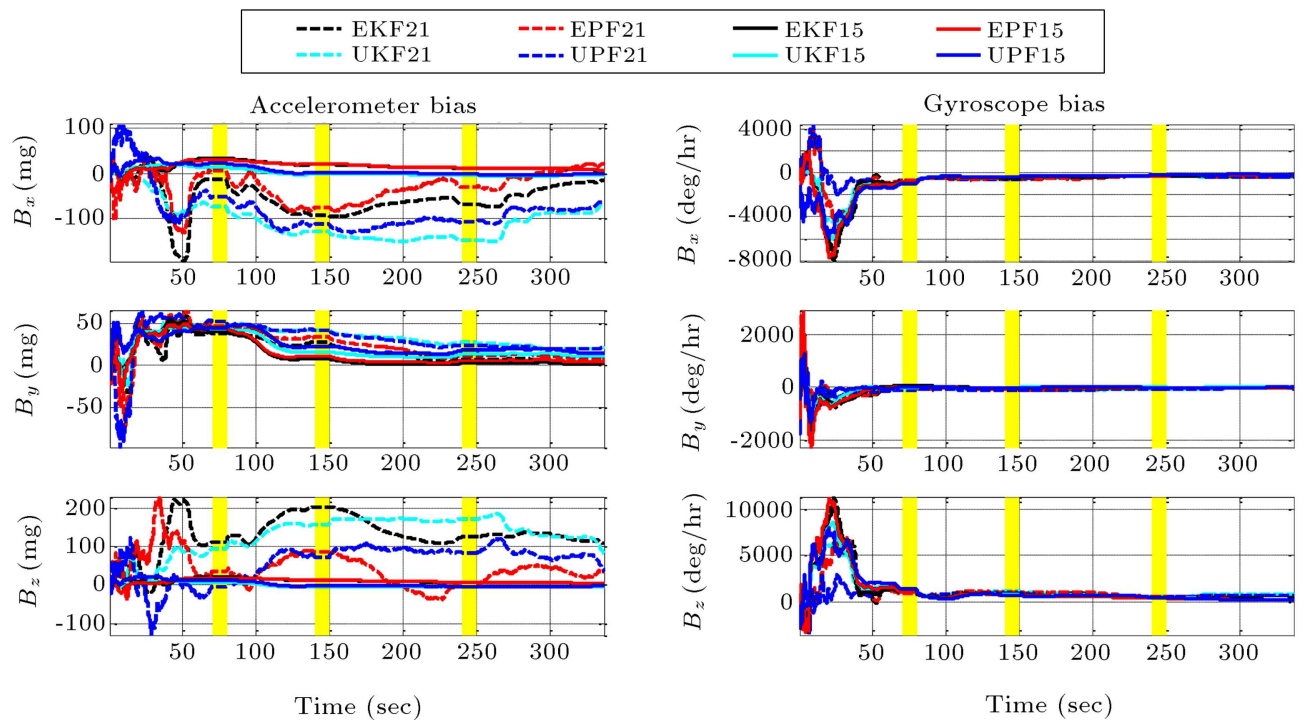

Figure 5. Gyro bias and accelerometer bias estimates for field test.

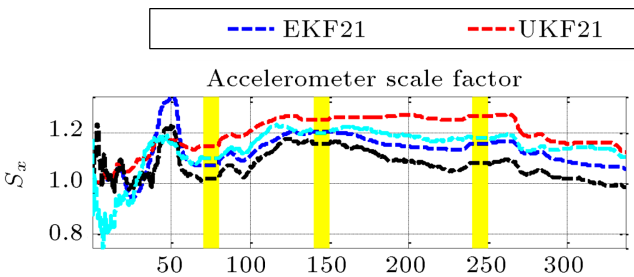

---- EPF21 ---- UPF21
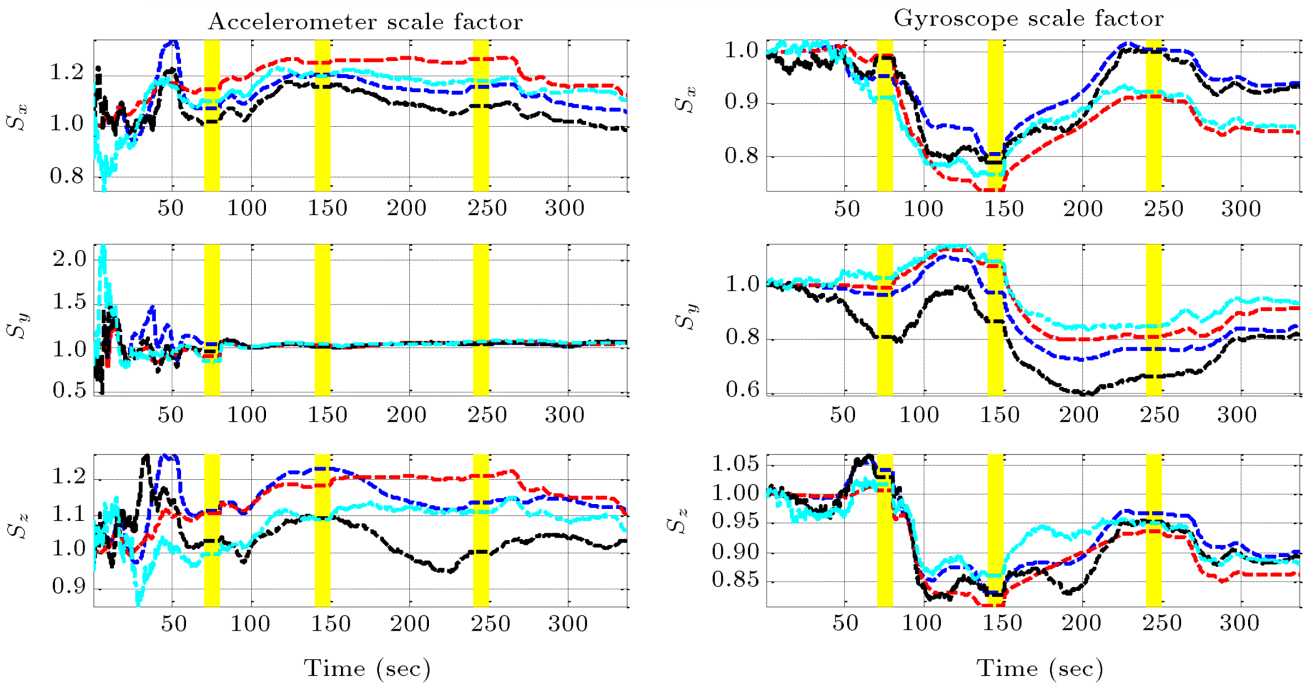

Figure 6. Gyro scale factor and accelerometer scale factor estimates for field test.

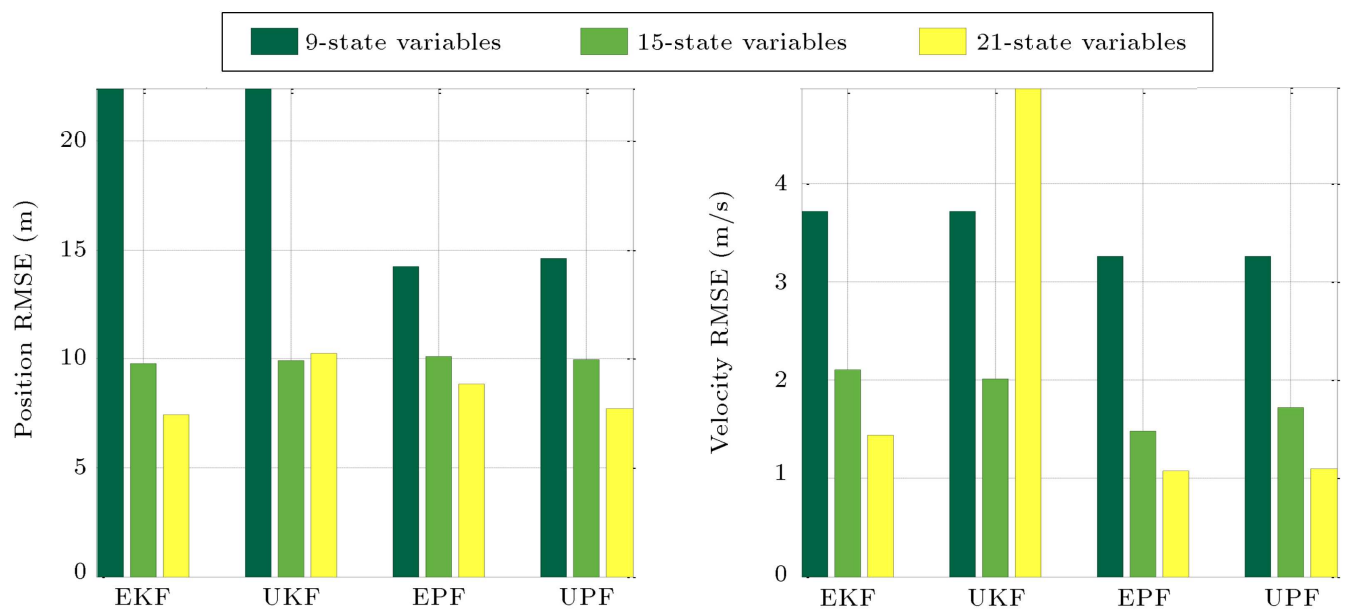

Figure 7. Position and velocity RMSE (Root Mean Square Error) for field test. 
Table 3. Root mean square error for field test.

\begin{tabular}{|c|c|c|c|c|}
\hline Filter & System model & $\begin{array}{c}\text { Position RMSE } \\
(\mathbf{m})\end{array}$ & $\begin{array}{c}\text { Velocity RMSE } \\
(\mathrm{m} / \mathrm{sec})\end{array}$ & $\begin{array}{c}\text { CPU time } \\
(\mathrm{sec})\end{array}$ \\
\hline \multirow{3}{*}{$\mathrm{EPF}$} & 9 -variable & 14.2218 & 3.2619 & $108.6703 \mathrm{~s}$ \\
\hline & 15 -variable & 10.0898 & 1.4777 & 130.4948 \\
\hline & 21-variable & 8.8541 & 1.0777 & 629.1208 \\
\hline \multirow{3}{*}{$\mathrm{UPF}$} & 9 -variable & 14.6036 & 3.2599 & 2089.0 \\
\hline & 15 -variable & 9.9574 & 1.7201 & 3834.9 \\
\hline & 21-variable & 7.6990 & 1.1022 & 7282.7 \\
\hline \multirow{3}{*}{ EKF } & 9 -variable & 22.4245 & 3.7213 & 14.4145 \\
\hline & 15 -variable & 9.7980 & 2.1030 & 15.1321 \\
\hline & 21-variable & 7.4562 & 1.4370 & 16.9261 \\
\hline \multirow{3}{*}{ UKF } & 9-variable & 22.4164 & 3.7203 & 147.8889 \\
\hline & 15 -variable & 9.9433 & 2.0140 & 252.4876 \\
\hline & 21-variable & 10.2458 & 4.9734 & 406.8974 \\
\hline
\end{tabular}

Table 4. Inertial sensors' characteristics.

\begin{tabular}{ccccc}
\hline & \multicolumn{2}{c}{ Accelerometer } & \multicolumn{2}{c}{ Gyroscope } \\
\hline Random walk & $\sigma_{\text {acc }}$ & $0.0005 \frac{\mathrm{g}}{\sqrt{\mathrm{Hz}}}$ & $\sigma_{\mathrm{gyr}}$ & $0.0017 \frac{\mathrm{deg} / \mathrm{s}}{\sqrt{\mathrm{Hz}}}$ \\
Bias time constant & $\tau$ & $60 \mathrm{~s}$ & $\tau$ & $100 \mathrm{~s}$ \\
Bias variation & $\sigma_{\text {biasacc }_{\text {a }}}$ & $50 \times 10^{-5} \mathrm{~g}$ & $\sigma_{\text {biasgyr }}$ & $0.35 \frac{\mathrm{deg}}{\mathrm{hr}}$ \\
& & & & \\
& $b_{\mathrm{acc}}^{x}$ & $2 \mathrm{mg}$ & $b_{\mathrm{gyr}}^{x}$ & $14 \frac{\mathrm{deg}}{\mathrm{hr}}$ \\
Constant offset & $b_{\mathrm{acc}}^{y}$ & $-2 \mathrm{mg}$ & $b_{\mathrm{gyr}}^{y}$ & $-14 \frac{\mathrm{deg}}{\mathrm{hr}}$ \\
& $b_{\mathrm{acc}}$ & $4 \mathrm{mg}$ & $b_{\mathrm{gyr}}^{z}$ & $10 \frac{\mathrm{deg}}{\mathrm{hr}}$ \\
Scale factor & & & & \\
Sample rate & $s_{\mathrm{acc}}$ & $300 \mathrm{ppm}$ & $s_{\mathrm{gyr}}$ & $150 \mathrm{ppm}$ \\
& $f_{s}$ & $100 \mathrm{~Hz}$ & $f_{s}$ & $100 \mathrm{~Hz}$ \\
\hline
\end{tabular}
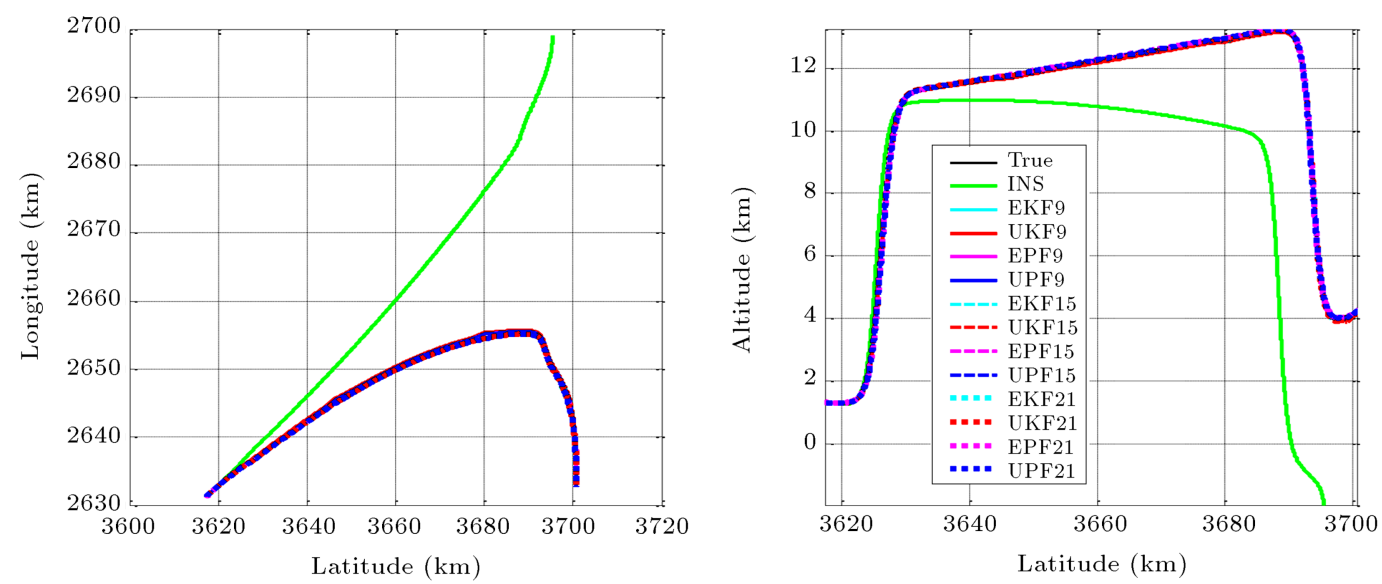

Figure 8. Position estimates for flight simulation. 
Table 5. Root mean square error for flight simulation.

\begin{tabular}{cccccc}
\hline Filter & $\begin{array}{c}\text { System } \\
\text { model }\end{array}$ & $\begin{array}{c}\text { Position RMSE } \\
(\mathbf{m})\end{array}$ & $\begin{array}{c}\text { Velocity RMSE } \\
(\mathbf{m} / \mathbf{s e c})\end{array}$ & $\begin{array}{c}\text { Angle RMSE } \\
(\mathbf{d e g})\end{array}$ & $\begin{array}{c}\text { CPU time } \\
(\mathbf{s e c})\end{array}$ \\
\hline \multirow{2}{*}{ EPF } & 9-variable & 336.6927 & 14.0956 & 2.1307 & 256.5904 \\
& 15-variable & 20.0499 & 2.6417 & 0.8998 & 312.7508 \\
& 21-variable & 20.0390 & 2.8496 & 1.0216 & 1451.5 \\
& & & & \\
\multirow{2}{*}{ UPF } & 9-variable & 328.2547 & 13.7649 & 2.1128 & 5000.5 \\
& 15-variable & 23.4743 & 3.0346 & 1.1245 & 9432.8 \\
& 21-variable & 20.9421 & 2.7604 & 1.0075 & 17448 \\
& & & & & \\
EKF & 9-variable & 493.8215 & 14.1492 & 2.1250 & 32.4638 \\
& 15-variable & 88.9628 & 3.0184 & 1.0496 & 35.1470 \\
& 21-variable & 88.9628 & 3.0184 & 1.0496 & 42.0423 \\
& & & & & \\
& 9-variable & 493.9893 & 14.1521 & 2.1251 & 332.6721 \\
& 15-variable & 88.4659 & 3.0046 & 1.0586 & 597.7958 \\
& 21-variable & 88.4659 & 3.0046 & 1.0586 & 1013 \\
\hline
\end{tabular}

vehicle experimental test and flight simulation test. In algorithms implementation, inertial sensors' characteristics and three types of system models with 9, 15, 21

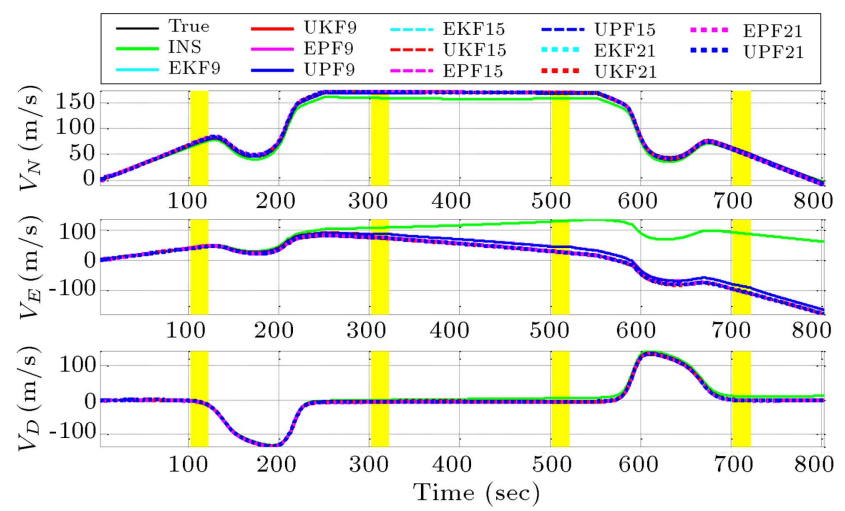

Figure 9. Velocity estimates for flight simulation.

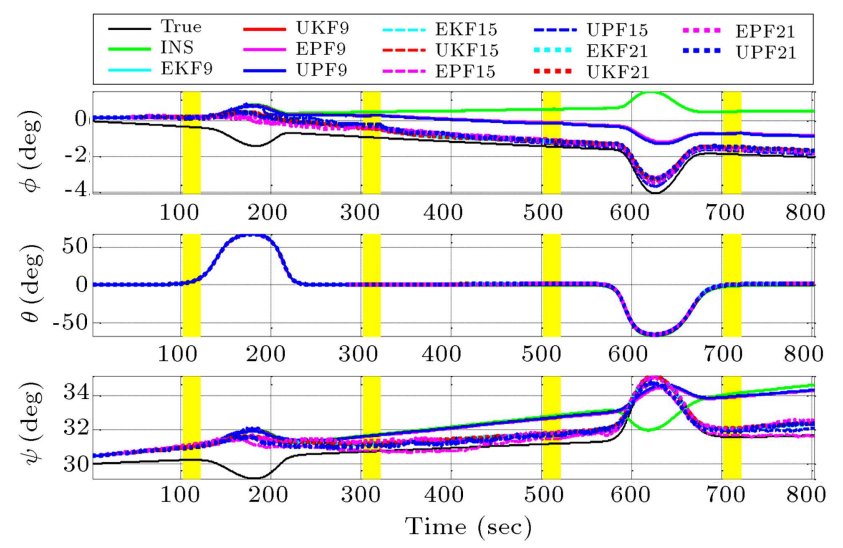

Figure 10. Euler angle estimates for flight simulation. state variables are considered. Some outcomes of the research are as follows.

Sensors output corrections led to highly accurate estimates in both dataset (i.e., the ground vehicle test and flight simulation). Estimation accuracies of 15variable and 21-variable system models are so much better than that of the 9 -variable system model in each filter. In addition, it is shown in the simulation that gyroscope biases are identified properly.

In each system model, four EKF, UKF, EPF, and UPF algorithms provide comparable results. According to the simulation results, EPF and UPF position estimates are more accurate than both EKF and UKF position estimates, while the velocity and attitude estimates are measured similarly by all four algorithms in each system model. According to the ground-vehicle test, estimates done by these four filters in each system model are close together, except for 9-variable system model case; EPF and UPF position estimates are more accurate than EKF and UKF position estimates are.

From a computational point of view, the EKF provided the lowest overall computational burden. Moreover, EPF and UKF algorithms can work in real time for 9-variable and 15-variable system models for both dataset.

Compared with the conventional PF algorithms, EPF and UPF can present accurate results with a small number of particles, as we chose 15 optimum particles for them. However, one drawback to EPF and UPF in drawing particles is that they depend on EKF and UKF estimates, which can be problematic in a serious non-Gaussian case. To enhance the estimates 

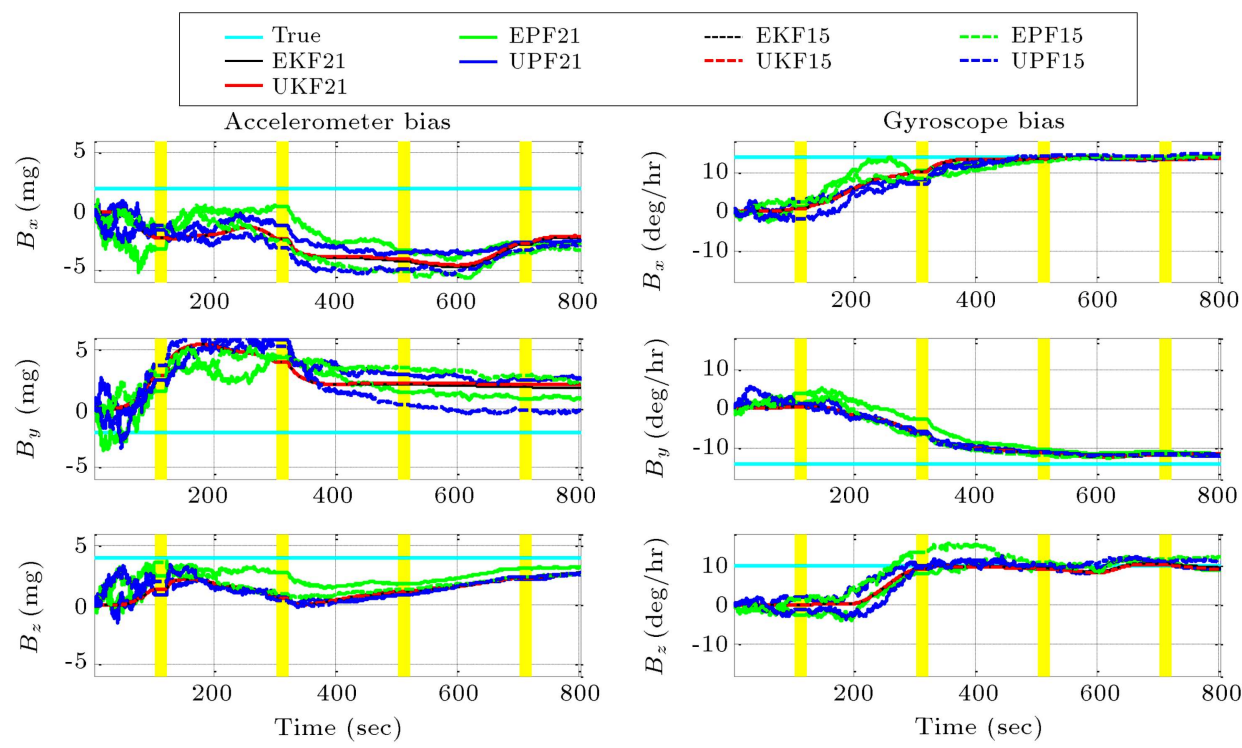

Figure 11. Gyro bias and accelerometer bias estimates for flight simulation.
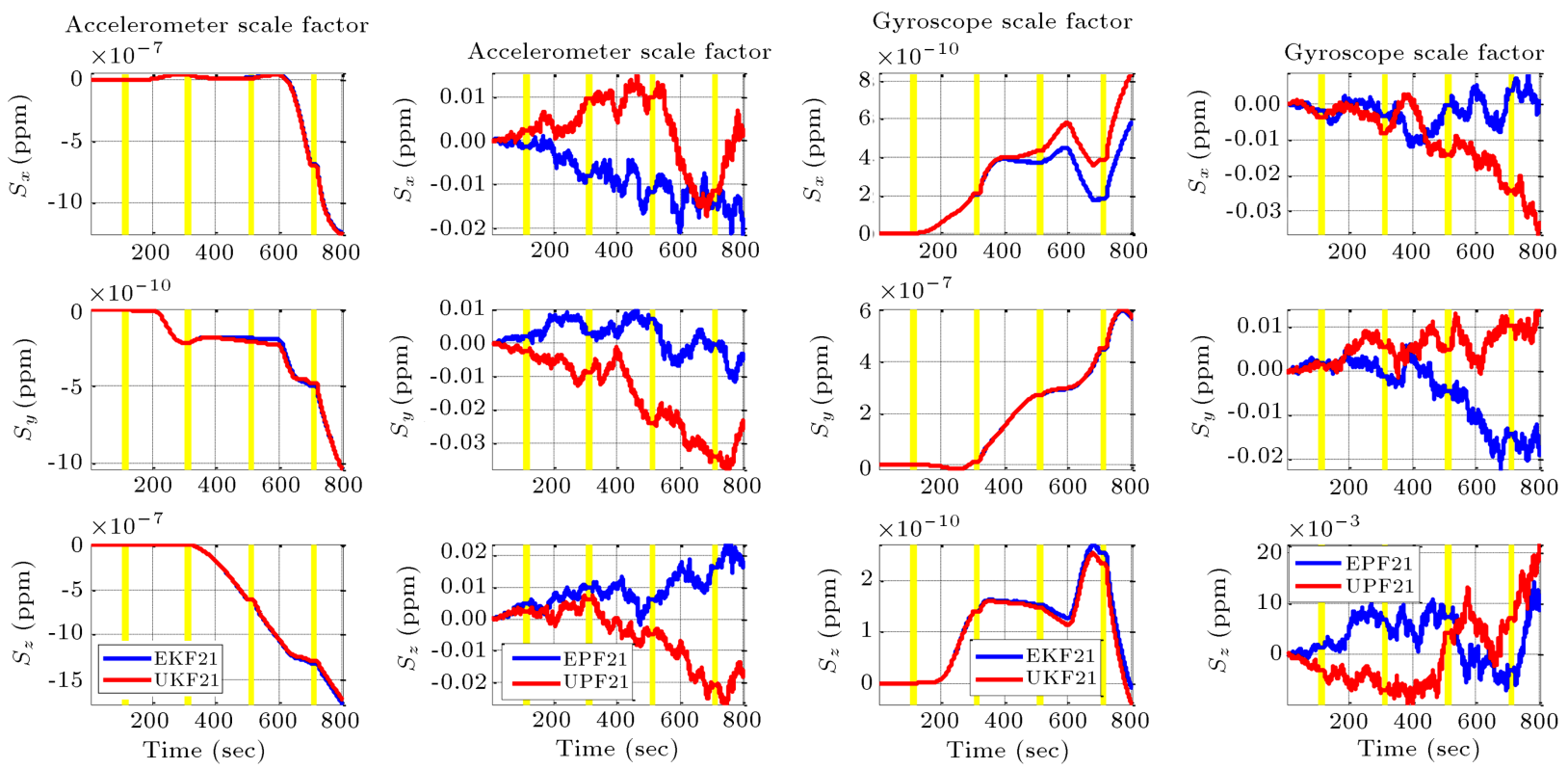

Figure 12. Gyro scale factor and accelerometer scale factor estimates for flight simulation.

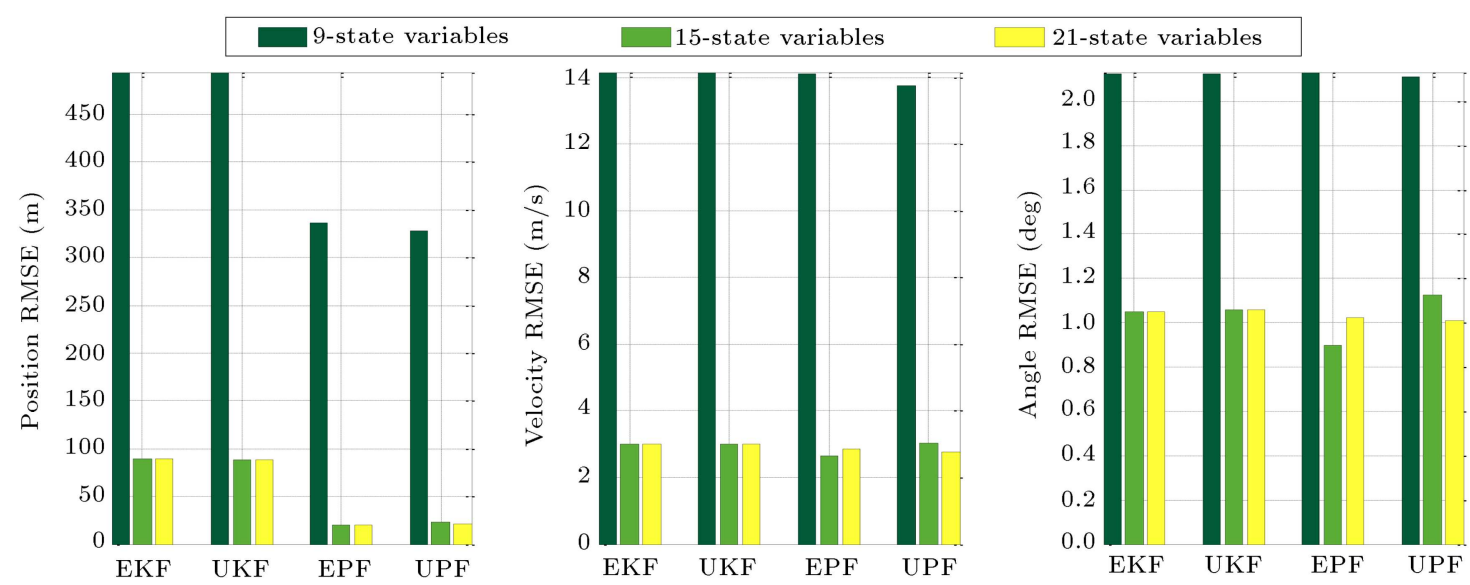

Figure 13. Position, velocity, and attitude RMSE (Root Mean Square Error) for flight simulation. 
accuracy, an implementation of a PF or KF, which can be updated along with other algorithms such as map matching, neural network or genetic algorithms, can be a next step within future efforts concerning the INSGPS integrated systems.

\section{References}

1. Wan, E.A. and Van Der Merwe, R. "The unscented Kalman filter for nonlinear estimation", in Adaptive Systems for Signal Processing, Communications, and Control Symposium 2000. AS-SPCC. The IEEE 2000, pp. $153-158$ (2000).

2. Chen, Z. "Bayesian filtering: From Kalman filters to particle filters, and beyond", Statistics, 182, pp. 1-69 (2003).

3. Gordon, N., Ristic, B., and Arulampalam, S., Beyond the Kalman Filter: Particle Filters for Tracking Applications, Artech House, London, 830 (2004).

4. Van Der Merwe, R., Doucet, A., De Freitas, N., and Wan, E. "The unscented particle filter", in Nips, pp. 584-590 (2000).

5. Wendel, J. and Trommer, G.F. "Tightly coupled GPS/INS integration for missile applications", Aerospace Science and Technology, 8, pp. 627-634 (2004).

6. Zhang, P., Gu, J., Milios, E.E., and Huynh, P. "Navigation with IMU/GPS/digital compass with unscented Kalman filter", in Mechatronics and Automation, 2005 IEEE International Conference, pp. 1497-1502 (2005).

7. Ryu, J.H., Gankhuyag, G., and Chong, K.T. "Navigation system heading and position accuracy improvement through GPS and INS data fusion", Journal of Sensors, 2016 (2016).

8. Yang, Y., Zhou, J., and Loffeld, O. "Quaternion-based Kalman filtering on INS/GPS", in Information Fusion (FUSION), 2012 15th International Conference on, pp. 511-518 (2012).

9. Lee, J.Y., Kim, H.S., Choi, K.H., Lim, J., Chun, S., and Lee, H.K. "Adaptive GPS/INS integration for relative navigation", GPS Solutions, 20, pp. 63-75 (2016).

10. Werries, A. and Dolan, J.M., Adaptive Kalman Filtering Methods for Low-Cost GPS/INS Localization for Autonomous Vehicles (2016).

11. Aggarwal, P., Syed, Z., and El-Sheimy, N. "Hybrid extended particle filter (HEPF) for integrated inertial navigation and global positioning systems", Measurement Science and Technology, 20, p. 055203 (2009).

12. Sun, F. and Tang, L. "Improved particle filter algorithm for INS/GPS integrated navigation system", in Mechatronics and Automation (ICMA), 2011 International Conference on, pp. 2392-2396 (2011).
13. Wang, X. and Ni, W. "An improved particle filter and its application to an INS/GPS integrated navigation system in a serious noisy scenario", Measurement Science and Technology, 27, p. 095005 (2016).

14. Zhou, J., Yang, Y., Zhang, J., and Edwan, E. "Applying quaternion-based unscented particle filter on INS/GPS with field experiments", ION GNSS 2011, pp. 3842-3855 (2011).

15. Li, K., Hu, B., Chang, L., and Li, Y. "Comparison of direct navigation mode and indirect navigation mode for integrated SINS/GPS", Transactions of the Institute of Measurement and Control, 38, pp. 3-13 (2016).

16. Gao, Y., Liu, S., Atia, M.M., and Noureldin, A. "INS/GPS/LiDAR integrated navigation system for urban and indoor environments using hybrid scan matching algorithm", Sensors, 15, pp. 23286-23302 (2015).

17. Li, J., Song, N., Yang, G., Li, M., and Cai, Q. "Improving positioning accuracy of vehicular navigation system during GPS outages utilizing ensemble learning algorithm", Information Fusion, 35, pp. 1-10 (2017).

18. Li, Z., Wang, J., Li, B., Gao, J., and Tan, X. "GPS/INS/Odometer integrated system using fuzzy neural network for land vehicle navigation applications", Journal of Navigation, 67, pp. 967-983 (2014).

19. Ren, M. "Vehicle positioning by map matching as feedback for ins/gps navigation system during gps signal loss", Ed: Google Patents (2014).

20. Britting, K.R. "Inertial navigation systems analysis" (1971).

21. Shin E.-H. and El-Sheimy, N. "Accuracy improvement of low cost INS/GPS for land applications", National Library of Canada [Bibliothèque nationale du Canada] (2003).

\section{Biographies}

Samira Kaviani obtained her BSc and MSc degrees in Mechanical Engineering from Amirkabir University of Technology and Sharif University of Technology, respectively, in years of 2008-2014. Her MSc thesis was titled by "Development of integrated navigation algorithm based on the integration of INS, GPS, altimeter using particle filters". At present, it is about two years that she has been involved in industry as a Mechanical Engineer.

Hassan Salarieh is a Professor of Mechanical Engineering Department at Sharif University of Technology. He received simultaneous degrees in Pure Mathematics and Applied Mechanics from Sharif University of Technology. Moreover, he obtained the ISME prize for the best PhD thesis in Applied Mechanics in May 2008. He has been selected as a Superior Researcher in the field of industrial projects in Sharif University of Technology 
in academic years of 2011-2012. Currently, His research is focused on fields of intelligent control, and filtering theory.

Aria Alasty is a Professor of Mechanical Engineering Department at Sharif University of Technology. He obtained different awards including Senate Gold Medallist for Distinguished PhD thesis in year 1996, and supervision of some projects and thesis in Mechanical Engineering. In addition, he was a Director, Chair, and member of Advanced Manufacturing research center, Academic Affairs, and CEDRA (Center of Excellence in Design Robotics and Automation), respectively. Currently, His research is focused on the fields of chaos control, intelligent machines, distributed parameter system, and robotics.

Mohammad Abediny received his all BSc, MSc, and $\mathrm{PhD}$ degrees in Mechanical Engineering from Sharif University of Technology in the years of 20032015. His main researches areas are in the fields of adaptive control, fractional calculus, and navigation. At the moment, he is a chief executive officer of SDRA. 